\title{
¿LÓGICA SIN VERDAD? *
}

\author{
Georg Henrik von Wright
}

RESUMEN. En este trabajo se discute la posibilidad misma de una lógica de las normas, al carecer estas de valor de verdad o falsedad. La tesis de fondo es que las nociones de consecuencia y contradicción lógica en términos de valores de verdad resulta persuasiva, pero es demasiado estrecha. Por ello, el trabajo intenta brindar una caracterización de las nociones de consecuencia y contradicción lógica que muestre tanto el modo en el que ellas dependen de la noción de verdad, como asimismo el modo en el que puede decirse que la trascienden.

Palabras clave: lógica de las normas, verdad, implicación, consecuencia.

\section{Logic without Truth?}

ABSTRACT. This paper discusses the very possibility of the construction of a deontic logic inasmuch as norms lack of truth or false value. The bottom thesis is that the notions of logical consequence and contradiction in terms of truth-values are quite compelling though still too narrow. Thus the paper attempts to offer a characterisation of the notions of consequence and logical contradiction that shows both how they rely on the notion of truth as well as how they transcend it.

Keywords: deontic logic, truth, implication, consequence.

* Fecha de recepción: 19 de abril de 2016. Fecha de aceptación: 1 de septiembre de 2016.

Traducción de Pablo E. NAVARRO. Revisión a cargo de Jorge L. RoDRíGUEZ. 


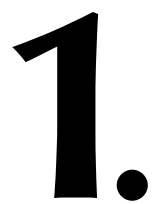

Una manera natural de explicar lo que significa que una cosa —una proposición, por ejemplo- se sigue lógicamente de otra es decir que, si la segunda es verdadera, la primera también tiene que ser verdadera. De modo similar, si se pregunta qué significa que una cosa contradiga, sea incompatible o inconsistente con otra, una respuesta natural es decir que ellas no pueden ser ambas verdaderas. Así, podría parecer que relaciones lógicas tales como las de consecuencia (implicación) y contradicción (incompatibilidad) de algún modo se apoyaran en la noción de verdad.

Sin embargo, no es difícil ofrecer ejemplos de cosas diferentes de proposiciones verdaderas o falsas entre las que se verifican, o parecen verificarse, relaciones lógicas. Los imperativos se cuentan entre ellas. «iAbra la puerta!», «iNo abra la puerta!». ¿No se contradicen lógicamente de algún modo estas dos órdenes, al menos si tienen por destinatario al mismo agente en la misma ocasión y se refieren a la misma puerta? «iAbra la puerta y cierre la ventana!». ¿No implica lógicamente esta orden conjuntiva a cada una de sus partes componentes? No obstante, no tiene sentido hablar de la verdad (o falsedad) de imperativos como los recién mencionados. Por ello, si la respuesta a las preguntas antes planteadas es afirmativa, las relaciones lógicas de contradicción e implicación se verifican también entre entidades distintas de proposiciones verdaderas o falsas.

Por otra parte, también hay oraciones en modo indicativo de las que resulta cuanto menos dudoso que expresen proposiciones verdaderas o falsas y, sin embargo, parece indudable que sus significados se encuentran entre sí en relaciones de contradicción o consecuencia lógica. Entre esas oraciones ocupan un lugar destacado aquellas que expresan (enuncian) normas, y también aquellas que expresan juicios de valor. En opinión de muchos filósofos, tales oraciones, como los imperativos y las exclamaciones, no dicen nada verdadero o falso. (Algunos filósofos han sostenido que las oraciones normativas y evaluativas son, de hecho, imperativos y exclamaciones con lo que podría denominarse un «disfraz proposicional»). Sin embargo, parece intuitivamente obvio que, por ejemplo, algunas normas se siguen lógicamente de otras normas, y también que una norma puede contradecir a, o ser lógicamente incompatible con, otra norma. Puede decirse que el reciente surgimiento de la nueva rama de estudios lógicos conocida como lógica deóntica confirma o incluso determina la corrección de esa intuición.

Por ello, la explicación sugerida de las ideas de consecuencia y contradicción lógica en términos de valores de verdad, aunque resulte natural o incluso persuasiva, parece demasiado estrecha. La pregunta sería: ¿puede ella extenderse más allá de los confines de la verdad y la falsedad? Al examinar aquí esta cuestión circunscribiré mi atención al discurso normativo. De manera un tanto provocativa, podría formular mi pregunta de la siguiente manera: ¿es realmente posible una lógica deóntica?

2. Se podría responder a esa pregunta de diferentes maneras.

Podría, por ejemplo, simplemente negarse que haya relaciones lógicas entre normas, sobre la base de que las normas no tienen valor de verdad. Esta fue la actitud de muchos de los primeros positivistas lógicos.

Podría considerarse como una versión más sofisticada de esta actitud la que sostiene que las relaciones lógicas que parecen verificarse entre normas, de becho se verifican 
entre entidades semejantes a las normas que son verdaderas o falsas. Esas entidades serían proposiciones relativas a que tal o cual norma existe ${ }^{1}$. Sus expresiones lingüísticas con frecuencia son idénticas a las de las propias normas.

Considerar que una lógica de normas es una lógica de proposiciones existenciales acerca de normas es una postura a cuyo favor yo mismo he argumentado en algunas publicaciones previas. Una debilidad de este enfoque es que las relaciones lógicas entre proposiciones existenciales no concuerdan muy bien con las relaciones lógicas que intuitivamente parecen verificarse entre genuinas normas. De todos modos, este enfoque de una lógica de normas merece todavía tomarse en serio.

Otros intentos de resolver el problema aceptan como un hecho que existen relaciones lógicas entre normas. Se podría entonces argumentar que la concepción de las normas como carentes de valor de verdad está equivocada. Las normas son verdaderas o falsas, podría decirse entonces. Esta posición sería, por ejemplo, sostenida por algunos propulsores de las llamadas doctrinas del derecho natural. La encuentro demasiado simple como para ser plausible.

Una posición más sofisticada trata de reconciliar la existencia de relaciones lógicas entre normas con la admisión de que las normas carecen de valor veritativo. Recientemente ALCHOURRÓN y MARTINO han propuesto una versión de esta posición ${ }^{2}$. Según ellos, relaciones como las de consecuencia y contradicción lógica subsisten también entre entidades distintas de aquellas que son verdaderas o falsas. La lógica, por así decirlo, se extiende más allá del dominio de la verdad. Esto era lo que yo también creía cuando desarrollé por primera vez un sistema de lógica deóntica. Pero lo hice ingenuamente, sin fundamentar.

Luego de un largo peregrinaje por las tierras inhóspitas de la lógica deóntica, volví sobre la cuestión en lo que entonces creí que sería mi última contribución a la disciplina, mi ensayo «Normas, verdad y lógica» ${ }^{3}$. En ese artículo, al igual que los primeros positivistas, negué que hubiera relaciones lógicas entre las normas porque ellas carecen de valor de verdad. Pero al mismo tiempo traté de mostrar cómo las normas genuinas pueden ostentar lo que podrían llamarse relaciones cuasi-lógicas, no definibles únicamente en términos de verdad y falsedad sino con la colaboración adicional de la noción de racionalidad. Todavía pienso que este movimiento constituyó una salida correcta del laberinto lógico en el que había estado vagando durante más de tres décadas. Pero

\footnotetext{
${ }^{1}$ Las proposiciones en cuestión con frecuencia se denominan «proposiciones normativas». Me temo que el término fue una invención mía. Mi idea era entonces que las proposiciones, por definición, son verdaderas o falsas, y que las normas, por carecer de valor de verdad, no son entidades proposicionales. Ahora encuentro más práctico usar el término «proposición» de manera diferente. Llamaría «proposición» al significado de cualquier oración gramaticalmente bien formada que satisface la siguiente condición: la oración que obtenemos al prefijarle la frase «es verdad que» está también gramaticalmente bien formada. Las típicas formulaciones normativas «Se debe...», «Debe ser el caso que...», etc., satisfacen esta condición. Por ello, consideraré a las normas que ellas expresan como (un tipo de) proposiciones.

2 C. AlChOURRÓn y A. MARTINO, «Logic without Truth» [Nota de los editores: al momento de escribir VON WRIGHT este trabajo, esa versión en inglés del artículo de ALCHOURRÓn y MARTINO aún no había sido publicada. Posteriormente apareció en Ratio Iuris (marzo 1990), vol. 3, 46-67].

3 Publicado en G. H. von Wright, Practical Reason. Philosophical Papers, vol. 1, Oxford, Basil Blackwell, 1983. Traducción al italiano de G. PeZzinI, con revisión técnica de A. A. MARTiNo, «Norme, verità e logica», Informatica e diritto, 9, 1983 (con un prefacio del autor a la traducción).
} 
ahora estoy dispuesto a conceder que esas relaciones cuasi-lógicas, dependientes de la noción de racionalidad, son genuinas relaciones de consecuencia y contradicción lógica. En consecuencia, he vuelto finalmente a mi opinión primigenia: que «la lógica trasciende la verdad».

Esta vez, no obstante, trataré de fundamentar esta posición. Más precisamente, lo que intentaré hacer es brindar una caracterización de las nociones de consecuencia y contradicción lógica que muestre tanto el modo en el que ellas dependen de la noción de verdad, como asimismo el modo en el que puede decirse que la trascienden.

3. Una primera y provisional manera de explicar la noción de consecuencia podría ser esta: que B se sigue de que A si, y solo si, si A entonces B. Una segunda manera alternativa sería: que B se sigue de que A si, y solo si, no: A pero no B. Si esas dos explicaciones de la noción de consecuencia son enteramente equivalentes es algo controvertible. Ellas son equivalentes si identificamos condicionalidad con implicación material. Porque entonces «Si A entonces B» se toma como si significara lo mismo que «no: A y no $B »$. Podría argumentarse, sin embargo, que hay un «remanente de significado» en el condicional que esa equiparación no alcanza a captar. Diré algo acerca de esto más adelante (infra, sección 9).

Ambas explicaciones son inteligibles y es fácil imaginar contextos en los que ambas o cualquiera de ellas podrían ofrecerse como respuesta a la pregunta sobre qué significa que una cosa se sigue de otra. De que se haya colocado este terrón de azúcar en agua se sigue que se disolverá. Esto niega que a pesar de que el azúcar se haya colocado en agua, ella no se disolverá.

Los «ingredientes lógicos» involucrados en una de estas explicaciones es la condicionalidad («si - entonces»), y en la otra la negación («no») y la conjunción («y»). No hay aquí ninguna mención a la verdad. Alguien podría decir que hay una «mención implícita» a ella: «si - entonces» sería una abreviatura de «si es verdad que..., entonces es también verdad que...». Pero esto no es siempre ni necesariamente el caso.

4. Aunque las explicaciones antes ofrecidas funcionan bastante bien para el uso coloquial de «se sigue de», son, al menos en cierto aspecto, insuficientes para nuestros propósitos. Ellas cubren un sentido «causal» y uno «lógico» de la expresión. De haber colocado el terrón de azúcar en agua se sigue que se disolverá. Pero a menos que consideremos a la solubilidad en agua como una propiedad definitoria de la sustancia azúcar, no diríamos que esto «se sigue lógicamente». Es más natural concebir a la solubilidad como una propiedad causal y, por tanto, contingente, del azúcar.

La noción de consecuencia lógica es, de algún modo, (una consecuencia) necesaria. Se puede tratar de captar esto diciendo algo semejante a que B se sigue lógicamente de que $\mathrm{A}$ si, y solo si, si $\mathrm{A}$ entonces necesariamente $\mathrm{B}$ o, alternativamente, necesariamente no: A y no B. En lugar de «necesariamente no» podemos decir también «imposiblemente».

No obstante, no es seguro que estas adiciones sean suficientes para distinguir la noción de consecuencia lógica de otros tipos de consecuencias. Podría pensarse que toda forma de consecuencia implica (alguna forma de) necesidad. De manera que la palabra «necesario» debería haber figurado en nuestras explicaciones desde el comien- 
zo. Se tendría entonces que distinguir la necesidad lógica de la causal y, tal vez, de otras formas de necesidad. ¿Cómo se haría esto? La misma noción de necesidad es oscura.

5. Alguien puede haber encontrado confuso mi uso de la partícula «que» en las expresiones «que $A$ » $\mathrm{y}$ «que $B$ ». Ello requiere pues de cierta aclaración.

Considérense los esquemas «Si A, entonces $\mathrm{B}$ » $\mathrm{y}$ «no: $\mathrm{A}$ pero no $\mathrm{B} »$. ¿Qué es lo que representan las letras «A»y «B»? No sería correcto decir que ellas representan las entidades entre las que se asume que subsisten relaciones lógicas. Lo que ellas representan es algo lingüístico, ciertas combinaciones de palabras que, cuando se las sustituye por esas letras, convierten a los esquemas en oraciones completas y bien formados del castellano. Estas entidades lingüísticas, a su vez, se encuentran en una relación semántica de representación con las entidades entre las que subsisten relaciones lógicas.

Entonces, ¿qué son estas entidades lingüísticas? (Esta es una pregunta que demasiadas veces se pasa por alto.)

No serviría decir simplemente que ellas son oraciones. La de oración es una categoría gramatical. Tampoco cualquier par de oraciones, cuando se las sustituye por «A $\mathrm{y} \ll \mathrm{B} »$, producirán una oración bien formada. Por ejemplo, no lo harían oraciones interrogativas o imperativas.

A veces se dice que $« A » y$ $« B »$ representan oraciones declarativas o descriptivas. Prefiero decir que ellas representan oraciones en modo indicativo. «Declarativa» y «descriptiva» no son lo que aquí entiendo por categorías gramaticales.

Las formulaciones normativas, i. e. oraciones que expresan o enuncian normas, se encuentran de manera típica en modo indicativo, pero no parecería natural llamarlas «declarativas» 0 «descriptivas».

A las oraciones en modo indicativo se les pueden prefijar frases como «es verdad que», «es necesario que», «está permitido que», etc. También se les pueden prefijar frases de carácter similar, expresivas de diversas actitudes epistémicas o doxásticas. Por ejemplo, «es sabido que» $\mathrm{o}$ «es dudoso que». Al prefijar estas frases a las oraciones en modo indicativo se obtienen nuevas oraciones indicativas (gramaticalmente correctas). Llamaré «cláusula "que"» a la parte de las nuevas oraciones consistente en la palabra «que» seguida por la oración original. Sobre las cláusulas «que» diré que nombran entidades a las que propongo llamar «proposiciones». Es entre estas entidades que pueden subsistir relaciones lógicas tales como las de consecuencia y contradicción.

Las proposiciones, así entendidas, son el «significado» de oraciones indicativas. Las descripciones, las declaraciones, las normas y las reglas son significados semejantes. En este sentido, las normas también son proposiciones.

Se dice a veces que los prefijos epistémicos y doxásticos «es sabido (creído, dudoso, etc.) que» se refieren a diversas actitudes proposicionales. En un sentido extendido, también puede decirse que los prefijos deónticos «es debido (permitido, no permitido) que» expresan actitudes proposicionales. $\mathrm{Y}$ entonces también «es necesario (posible, imposible) que» e incluso «es verdadero (falso) que».

6. Considérese el esquema «Si A, entonces $\mathrm{B} »$. Hay al menos dos tipos de casos en los que las letras no representan oraciones indicativas. Uno es cuando «B» repre- 
senta una oración imperativa y «A» una indicativa. Como, por ejemplo, en «Si el perro ladra, no corras». Este tipo de condicionales no nos interesa aquí. El segundo tipo es conocido como condicional contrafáctico o subjuntivo. Algo debe decirse sobre ellos.

Quizás usualmente los condicionales contrafácticos se refieren a relaciones causales. «Si el terrón de azúcar se hubiese colocado en agua, se habría disuelto». Pero bien pueden también referirse a relaciones de consecuencia lógica. «Si Russell no hubiese estado casado, habría sido soltero».

Cuando la frase «Russell no hubiese estado casado» aparece luego de «si», no es una oración indicativa — sí lo es cuando aparece independientemente- Ni siquiera es una oración completa del castellano, como tampoco lo es «habría sido soltero».

Adoptaré el enfoque de que «si Russell no hubiese estado casado, habría sido soltero» es una variante idiomática de «si Russell no estaba casado, era soltero, pero Russell estaba casado». La relación de consecuencia lógica se verifica entre (las proposiciones) que Russell no estaba casado y que era soltero. Esto es así independientemente de si Russell estaba, de hecho, casado o no.

En consecuencia, la sugerencia sería que un condicional contrafáctico es una conjunción disfrazada. Su primer término es un condicional cuyo antecedente y consecuente son oraciones indicativas. Su segundo término es la oración que constituye la negación del antecedente del condicional.

$\mathrm{Si}$ «Si A entonces $\mathrm{B} »$ es un condicional contrafáctico, no puede «traducirse» como «no: A y no B». Esta segunda expresión no es entonces una oración bien formada sino un simple sinsentido.

7. Como se ha insinuado antes, una manera tradicional de explicar qué significa (la proposición) que B se sigue lógicamente de (la proposición) que A es decir que si la segunda es verdadera, la primera necesariamente es, tiene que ser o no puede dejar de ser, también verdadera.

¿Cuánto añade esta ampliación, que incorpora la noción de verdad, a la fuerza explicativa del esquema «Si A entonces necesariamente $\mathrm{B} »$ como explicación propuesta de la noción de consecuencia lógica? La misma pregunta puede plantearse respecto de la ampliación de nuestro segundo esquema a «es necesariamente verdadero que es verdadero que $A$ pero no es verdadero que $B »$.

Una respuesta podría ser que las ampliaciones no añaden nada en absoluto al poder explicativo de los esquemas. Porque de acuerdo con un punto de vista muy extendido, si «X» representa una oración indicativa, entonces «es verdad que $\mathrm{X}$ » no dice nada más allá de «X» mismo.

Incluso concediendo que esta equiparación no está exenta de discusión, la mera introducción de la noción de verdad no ilumina demasiado la idea de consecuencia. Otras nociones podrían resultar más útiles. Una de ellas es la de «aceptación» y su opuesta, la de «rechazo». Se diría entonces, por ejemplo, que B se sigue lógicamente de que A significa que si se aceptara lo primero, se estaría «por consistencia» comprometido, obligado a aceptar o no podría rechazar, también lo segundo.

Reemplazamos nuestros esquemas explicativos iniciales por los dos siguientes: que B se sigue lógicamente de que A significa (a) que se debe aceptar que B si se acepta que 
A o, alternativamente, (b) que se debe rechazar la conjunción de que A con la negación de que $B$.

Los ingredientes conceptuales de los dos esquemas son: condicionalidad ( $«$ si - entonces»), necesidad («tener que»), conjunción («y») y aceptación y rechazo.

8. ¿Qué es aceptar o rechazar una proposición? Aceptar una proposición, podría decirse, es «aferrarse» a ella, excluirla del debate y de la duda, usarla como un estándar para medir (juzgar) la aceptabilidad o la no aceptabilidad de otras proposiciones. Estas aclaraciones, si bien vagas, se aplican no solamente a proposiciones con valor de verdad sino también, por ejemplo, a las normas. Las normas, aun cuando no sean verdaderas o falsas, son sin duda objeto de aceptación o rechazo. Se puede debatir sobre ellas, y como resultado de ese debate nuestras actitudes hacia ellas pueden cambiar.

Aceptar una proposición a menudo significa creer en ella, o incluso conocerla. La creencia en una proposición normalmente significa creer en la verdad de la proposición. Esta forma de aceptación de las proposiciones no se aplica a las normas (genuinas).

Con frecuencia, pero no siempre, se puede argumentar, dar razones o fundamentos a favor de la aceptación de una proposición. Estos fundamentos constituyen un conjunto heterogéneo. Cuando se acepta una proposición como verdadera, los fundamentos ofrecidos en apoyo de su aceptación tienen que ver con cosas tales como el testimonio de nuestros sentidos, la confianza en la autoridad de otros individuos, o inferencias a partir de otras proposiciones ya aceptadas. La aceptación no «convierte» a una proposición en verdadera, pero declarar verdadera a una proposición es destacarla para el papel que las proposiciones aceptadas desempeñan en nuestra vida y en nuestras relaciones con otras personas.

Parece existir la siguiente notable asimetría entre las ideas de aceptación y rechazo (de proposiciones). Las proposiciones son rechazadas porque se contradicen o resultan de algún otro modo irreconciliables con otras proposiciones aceptadas. El rechazo, entonces, presupone y es secundario respecto de la aceptación. Por ejemplo, rechazo la proposición de que Russell era soltero porque acepto como un hecho que estaba casado. Si dudase acerca de si estaba o no casado, debería saber cómo establecer la verdad sobre la cuestión — buscando en libros o preguntándole a alguien cuya autoridad acepto-

También rechazo la conjunción de que Russell era casado y era soltero. Aquí el fundamento del rechazo es, por así decirlo, interno respecto de la proposición (molecular). No necesito aceptar que Russell no estaba casado o que no era soltero a fin de rechazar esa proposición. Ningún hecho acerca de Russell es relevante para mi rechazo. ¿Debería no obstante decir que la rechazo por ser «contraria a la verdad»? Podría decir eso, pero decirlo tendería a desdibujar la diferencia entre mi fundamento para rechazar esa proposición y para rechazar la proposición de que Russell era soltero. ¿Por qué, entonces, Russell no era un soltero casado? Porque no puede haber tal cosa. Imaginar la posibilidad sería contrario a la razón (más que contrario a la verdad); por tanto, no podemos aceptarla. La noción de un soltero casado no tiene sentido, excluimos la forma de expresión «soltero casado» como contraria a un estándar aceptado de discurso significativo. Es debido a esta actitud hacia la proposición que también decimos que, si Russell estaba casado, se sigue lógicamente que no era soltero. $\mathrm{O}$, alternativamente, que si era soltero, necesariamente no estaba casado. 
Lo que trato de decir es que no es porque la negación de una proposición se siga lógicamente de la otra, o que no es porque las dos proposiciones se contradicen lógicamente, que rechazamos su conjunción. Es el rechazo de la conjunción como algo contrario a la razón o como un sinsentido lo que confiere a sus miembros estas particulares relaciones que llamamos, respectivamente, consecuencia y contradicción lógica. La lógica no nos «fuerza» al rechazo; deberíamos más bien decir que es nuestro rechazo lo que «confiere» a las proposiciones el orden al que denominamos «lógico».

La necesidad lógica no es una clase de super-verdad, sino un reflejo de actitudes que adoptamos hacia el modo en que las proposiciones están mutuamente relacionadas desde el punto de vista de su aceptabilidad y rechazo. A fin de dar sentido a las nociones de consecuencia y contradicción lógica no necesitamos asumir que las proposiciones así relacionadas son verdaderas o falsas. Lo que se debe asumir, no obstante, es al menos que podemos dar previamente sentido a las operaciones de conjunción y negación que les aplicamos. Como se verá con claridad enseguida, esta condición no es una trivialidad.

9. Veamos ahora cómo puede aplicarse lo que hemos dicho a las normas.

Las normas se expresan en oraciones indicativas a los efectos de que ciertas cosas, normalmente acciones humanas, sean obligatorias, permitidas y prohibidas, i. e. deban, puedan o no deban hacerse.

Que la conjunción y la negación se aplican a las normas significa que las oraciones que obtenemos al unir oraciones que expresan normas por medio de la partícula «y» (o «pero») y al prefijar a ellas la palabra «no» también expresan normas. La persona a quien se dirigen las oraciones así formadas tiene que saber lo que es obligatorio, permitido, o prohibido si sabe qué normas expresan las oraciones simples.

«Y»y «no» son a menudo llamadas conectivas veritativas. Como tales, no obstante, solo se aplican a proposiciones que tienen valor de verdad. En tal caso el significado de la conjunción y la negación es claro y fácil de explicar.

También está claro y fuera de discusión que «y» y «no» se aplican a proposiciones a los efectos de que hay (existen) tales y cuales normas. Esas proposiciones tienen valor de verdad. Ellas a menudo, quizás normalmente, se expresan mediante (instancias de) las mismas (clases de) oraciones que las propias normas (cfr. supra, sección 1).

Si decir que debe ser que A $y$ debe ser que B es decir que hay una norma a efectos de lo primero y también una norma a efectos de lo segundo, entonces esto será verdadero o falso según sea el caso. Pero eso no es expresar una norma. Tomar consciencia de ello debería hacernos preguntar si la operación de conjunción tiene alguna aplicación a las normas. No estoy sugiriendo que la respuesta tiene que ser «No». Pero tampoco es claro que pueda ser «Si».

La conjunción de proposiciones con valor de verdad es en sí misma una proposición con valor de verdad. Se podría pensar que, de manera análoga, una conjunción de normas tiene que ser también una norma.

Esta sugerencia es fácil de seguir para el caso simple recién mencionado. Decir que debe ser que A y que debe ser que B puede ser — de hecho muchas veces es- otra manera de decir que debe ser que A y B. Esta última oración puede ser usada para 
expresar una norma, al igual que puede ser usada para expresar que hay una norma a tal efecto. Podría llamársela una norma de «contenido conjuntivo».

Para casos más complicados la sugerencia no es tan fácil de seguir. ¿Qué norma o normas responden a la conjunción de dos o más permisiones, o a la conjunción de obligaciones y permisiones? Estas preguntas también tienen que tener una respuesta satisfactoria si ha de ser posible una lógica deóntica como genuina lógica de normas. Pero no trataré de responder a ellas aquí.

Hasta aquí hemos hablado de conjunciones de normas. ¿Qué hay de la negación? ¿Qué significa decir que no es obligatorio que A? Podría significar que no hay ninguna norma que declare obligatorio que A. Este es un enunciado verdadero o falso. Pero también podría ser una manera de declarar permitido que no A. Esta es una genuina norma. La llamaré la norma-negación de la norma que declara obligatorio que A. Así, la negación de una norma que impone una obligación es una norma que confiere un permiso.

Mediante un argumento similar se puede mostrar que la norma-negación de una norma permisiva es una norma de obligación. «No está permitido que A» puede expresar o bien un enunciado verdadero o falso a los efectos de que no se ha conferido un cierto permiso, o bien puede expresar una norma a los efectos de que es obligatorio que no A, i. e. está prohibido que A.

Estas observaciones sobre la aplicación de la negación a las normas están en armonía con nuestras intuiciones conceptuales y con el uso del lenguaje.

En consecuencia, concediendo que las conectivas «y» y «no» se aplican también a las normas, podemos ilustrar mediante un ejemplo sencillo cómo esto podría habilitarnos a decir que una norma se sigue lógicamente de otra.

Considérese la combinación de palabras «debe ser que A y debe ser que no A». Como norma esto dice que debe ser que A y que no A. ¿Debemos rechazar esto? ¿Y qué significaría rechazarlo?

Una norma no puede ser rechazada por ser contraria a la verdad (i. e. por decir algo falso). Pero puede ser rechazada por ser irracional, contraria a la razón. ¿Por qué? Esta pregunta solo puede responderse sobre la base de consideraciones relativas al uso y propósito de la actividad de dictar normas.

Las normas normalmente pretenden asegurar un cierto orden de cosas, que puede obtenerse a través de la acción humana o de la abstención de la acción. La autoridad que hace obligatorio que A quiere que sea el caso que A. También con frecuencia está preparada para tomar ciertas medidas para lograr que aquellos a quienes la norma se dirige cumplan con su deseo. Por tanto, quien hiciera obligatorio que A y no A se comportaría como si desease que sea el caso que A y que no A. Pero eso, diríamos normalmente, no puede ser. Ninguna medida destinada a asegurar que se cumpla con tal «deseo» podría ser eficaz. Por tanto, tampoco tiene sentido dictar esa norma. En «nombre de la razón» la rechazamos, nos rehusamos a incluirla en cualquier conjunto o código normativo razonable.

A partir de esto podemos pasar a hablar sobre consecuencia lógica. Dado que rechazamos la conjunción de las normas, podemos decir de cualquiera de ellas que su 
norma-negación se sigue de la otra norma. Por ejemplo: de la norma de que debe ser que A se sigue lógicamente la negación de la norma de que debe ser que no A, i.e. la norma de que puede ser que $A$. De manera similar, de la norma de que debe ser que no A se sigue lógicamente que puede ser que no A. En general, de la norma que declara algo como obligatorio se sigue lógicamente una norma que permite esa misma cosa. «La obligación ("deber") implica la permisión ("poder")».

En lugar de decir que rechazamos la conjunción de las dos normas podríamos también decir que ellas se contradicen, o que una es inconsistente con la otra. Pero no es porque ellas sean contradictorias o inconsistentes que las rechazamos, sino que se las considera contradictorias porque rechazamos su conjunción como «contraria a la razón».

Debe advertirse incidentalmente que alguien puede sentir una cierta resistencia a pasar, de decir que dos normas son contradictorias, a decir que la negación de una se sigue lógicamente de la otra. Pero pienso que esta resistencia tiene sus raíces en una confusión. Del hecho de que hay una norma que declara algo como obligatorio no necesariamente se sigue que también hay (existe) una norma que permite ese algo. Esto se sigue o no dependiendo de cuál sea el criterio que adoptemos para la existencia de normas. $\mathrm{Si}$, por ejemplo, exigimos que una norma sea «promulgada» en palabras para que exista, no se sigue que, si hay una norma de obligación, hay también una norma permisiva. Pero aquello que debe ser puede, no obstante, también ser.

Una parte de la razón por la que rechazábamos la conjunción de las dos normas era que la conjunción de sus «contenidos», que A y que no A, era algo que nunca podía ser verdadero. Había, entonces, un doble rechazo involucrado: la conjunción de las normas se rechazaba como contraria a la razón porque demandaba algo que rechazamos como contrario a la verdad.

Pero, ¿debe ser esto así? ¿Debemos rechazar la conjunción de cualquier proposición con su negación? No hay ningún «deber» aquí, por «detrás» del «deber» que surge del propio rechazo.

Si no rechazamos la conjunción de que A y que no A, tampoco tenemos razón para rechazar la conjunción de las dos normas que requieren tanto que $\mathrm{A}$ como que no $\mathrm{A}$. De hecho, esas normas no son tales que nunca hemos escuchado de ellas, si bien tal vez lo hacemos con mayor frecuencia en el mundo de la ficción y la fantasía que en el de los hechos. Existe un cuento sobre un rey que ordenó a la hermosa Kraka que lo visitase vestida y desnuda. ¿Qué hizo la inteligente mujer? Se cubrió con una red de pesca y fue a ver al rey. ¿No estaba vestida? ¿No estaba también desnuda? Tomemos estas preguntas en serio y pensemos. No es en absoluto antinatural, «contrario a la razón» o «contrario a la verdad», decir que Kraka estaba, a la vez, vestida y desnuda. Tampoco nos resultaría antinatural decir que ella no estaba ni vestida ni desnuda ${ }^{4}$.

En una lógica deóntica que no rechace conjunciones de normas de obligación con contenidos «opuestos» ya no será el caso que la negación de la norma de que debe ser el caso que no A se siga lógicamente de la norma de que debe ser el caso que A. Manteniendo el mismo significado que le asignamos antes a la negación de una norma

${ }^{4}$ Cfr. Rabelais, Panurge, Ch. XXXVI, sobre el consejo del filósofo respecto de si Pantagruel debía casarse. 
de obligación se seguiría que en esta lógica deóntica la obligación no implica la permisión. Podemos aceptar esto y decir, por ejemplo, que Kraka no tenía permitido visitar vestida al rey a pesar de que estaba obligada a ello. En el caso considerado no habría nada extraño en ese uso del lenguaje.

En un mundo en el que las cosas estuvieran siempre, o la mayor parte del tiempo, en continuo cambio, o en el que la mayoría de las características de las cosas se fusionasen sin límites precisos, podrían tener que aceptarse enunciados semejantes a contradicciones tanto en la descripción de cómo son las cosas como así también para decir cómo deberían o podrían ser.

Algunos filósofos han sido de la opinión de que el mundo en el que vivimos es ciertamente así, y que es solo la trama gruesa de la red con la que para la mayoría de nuestros propósitos lo conceptualizamos lo que hace que las cosas parezcan diferentes. Esta opinión, sin embargo, me parece que se funda en una idea indebidamente «realista» de lo que «es» el mundo independientemente de su conceptualización.

Podría decirse que en un mundo «lleno de contradicciones» no habría ningún «orden lógico» de las cosas. Pero entonces debería advertirse que las conjunciones aceptadas de proposiciones y sus negaciones no son contradicciones en el sentido «clásico», que requiere el rechazo de uno de sus miembros si el otro es aceptado. ¿No podría haber una lógica, un orden lógico, entre tales conjunciones «contradictorias» en sentido no clásico? La respuesta es «Si». Las diversas lógicas agrupadas bajo el rótulo de «paraconsistentes» constituyen un ejemplo. Pero, por lo que entiendo, también en ellas la noción de consecuencia lógica es «clásica», i. e. basada en el rechazo de ciertas conjunciones por ser, en el sentido clásico, contradictorias.

10. He tratado de mostrar, usando la lógica deóntica como ejemplo, cómo la noción de consecuencia lógica puede también aplicarse a proposiciones sin valor de verdad. Constituye una particularidad del ejemplo utilizado el que un fundamento para rechazar cierta conjunción como contraria a la razón está dado por el rechazo de otra conjunción como contraria a la verdad. Así, en nuestro ejemplo, las relaciones de consecuencia entre proposiciones sin valor de verdad presuponen relaciones de consecuencia entre proposiciones con valor de verdad. Si esto tiene que ser así, i. e. si este es un presupuesto general de las relaciones de consecuencia (y de otras relaciones lógicas) entre proposiciones sin valor de verdad, es una cuestión que no discutiré aquí. Me inclino a pensar que la respuesta es afirmativa. Si esto es correcto, podría decirse que relaciones lógicas tales como las de consecuencia y contradicción se verifican primariamente entre proposiciones con valor de verdad y solo en un sentido secundario entre proposiciones que no satisfacen esa condición.

11. Voy a concluir con algunas observaciones sobre ciertas conocidas anomalías o «paradojas» inherentes a la identificación de la noción de implicación o consecuencia lógica con el rechazo por inconsistente de la conjunción de una proposición con la negación de otra proposición.

Supóngase que, por alguna razón, la conjunción de dos proposiciones, que A y que $\mathrm{B}$, es rechazada como contradictoria (contraria a la razón). En tal caso, la adición de un tercer miembro a la conjunción, digamos la proposición que $\mathrm{C}$, presumiblemente también tendría que ser rechazada. Y entonces, de acuerdo con nuestra definición, la 
proposición que no $\mathrm{C}$ se sigue lógicamente de la proposición conjuntiva que A y que $\mathrm{B}$. En virtud de un argumento similar, la proposición que $C$ también se sigue lógicamente de la conjunción.

Esta es otra manera de plantear la bien conocida idea de que a partir de una contradicción se sigue lógicamente cualquier proposición. Esto puede parecernos extraño. No obstante, la aceptación de esta rareza se ha vuelto casi corriente en la lógica moderna. La observación también se toma como fundamento para decir que la ocurrencia de una contradicción en un «sistema» de proposiciones lo «hace explotar» o lo «trivializa». Esto, se dice, es lo «malo» de las contradicciones.

Vale la pena preguntarse si se podría modificar nuestra definición de consecuencia a fin de evitar lo extraño de tener que decir que de una contradicción se sigue cualquier proposición (evitar esta expresión no volvería «aceptables» a las contradicciones; ellas por definición, podría decirse, son cosas que rechazamos por ser contrarias a la razón y a la verdad).

Lo que tiene de extraño decir que no $\mathrm{C}$ se sigue de la conjunción antes mencionada es que ella ya es una contradicción antes de (sin) la adición de que C. Si no hubiese sido ya contradictoria, pero se hubiese vuelto (convertido en) contradictoria por la adición de que $\mathrm{C}$, entonces hubiese sido el caso —-más allá de cualquier cuestionamiento sobre su «rareza»— que la proposición que no $C$ se sigue lógicamente de la conjunción de las otras dos proposiciones.

Llamamos auto-contradictorias a ciertas proposiciones. Por ejemplo, que Russell era un soltero casado. Podría preguntarse si una proposición auto-contradictoria es una conjunción «disfrazada» de proposiciones que se contradicen. Que Russell era un soltero casado significa que Russell era soltero y que Russell era casado. Que Russell era soltero significa que no era casado. Por consiguiente, que Russell era un soltero casado significa que Russell era casado y que Russell no era casado.

Debería advertirse que aquí debemos decir «significa» y no «se sigue de» $\mathrm{o}$ «implica». Porque decir que la proposición que Russell era casado implica que no era soltero ya presupone que rechazamos la conjunción que Russell era casado y que Russell era soltero. La naturaleza auto-contradictoria de la proposición que Russell era un soltero casado es una «cuestión de significado», no una cuestión de consecuencia lógica. Que sea lo primero significa que excluimos del uso correcto del lenguaje una cierta combinación de palabras («casado soltero»). Por tanto, la idea que esa combinación «pretende» expresar es «contraria a la razón».

Si es auto-contradictorio que $\mathrm{B}$, entonces también es contradictorio que A y que B. Si la noción de consecuencia es definida simplemente como la inconsistencia de una proposición con la negación de otra, tendríamos que decir que la negación de que $\mathrm{B}$ se sigue lógicamente de que A. La negación de una contradicción a veces se denomina tautología. (Si una contradicción es una proposición necesariamente falsa, su negación es una proposición necesariamente verdadera). La observación anterior significa entonces que cualquier tautología se sigue lógicamente de cualquier proposición arbitraria. Esto también puede considerarse como un sentido vacuo de consecuencia lógica al que desearíamos «eliminar» de algún modo. Para lograr esto sugiero la siguiente definición modificada de implicación o consecuencia lógica: 
Una proposición se sigue lógicamente de otra si, y solo si, la conjunción de la segunda con la negación de la primera es inconsistente $y$ esta inconsistencia es independiente del hecho — si es un hecho- de que la segunda proposición es en sí misma inconsistente y también del hecho - si es un hecho- de que la primera es tautológica ${ }^{5}$.

¿Qué significa aquí «independiente»? Podría tratar de responderse así: significa que incluso si una proposición no fuese contradictoria o la otra tautológica, la conjunción de una con la negación de la otra sería una inconsistencia.

Esta respuesta puede, no obstante, ser considerada poco esclarecedora. Genera la siguiente pregunta: ¿qué clase de «hipótesis» estamos considerando cuando decimos que algo sería el caso si una proposición que es contradictoria no lo fuese, o si una proposición que es tautológica no lo fuese? El significado de estos contrafácticos no es claro.

Me parece que una respuesta satisfactoria debe hacer referencia al modo en que demostramos, establecemos, llegamos a conocer el carácter inconsistente de la conjunción de una proposición con la negación de la otra. «Independiente» en la definición sugerida significa «demostrabilidad independiente». ¿Qué es esto? Trataré de aclararlo mediante algunos ejemplos.

Considérese la conjunción de dos proposiciones, que A y que $\mathrm{B}$, y uno de los miembros de esa conjunción, digamos que $\mathrm{A}$. La conjunción de la conjunción y la negación de ese miembro, i.e que $\mathrm{A} \& \mathrm{~B} \& \sim \mathrm{A}$, es una contradicción. Ello es así con independencia de si la conjunción de que $\mathrm{A}$ y que $\mathrm{B}$ es o no es en sí misma una contradicción, y también con independencia de si $\mathrm{A}$ es o no es una tautología. Por eso es que $\mathrm{A} \& \mathrm{~B}$ implica que $\mathrm{A}$. Esta es una instancia de una «ley de implicación» general, según la cual una conjunción implica a cada uno de sus miembros.

Ahora substituyamos « A» por «B». Que A\& A es una contradicción. ¿Esta conjunción implica también a sus miembros, por ejemplo, implica que A? Que A\& A\& A también es una contradicción. Esto es (ya) así porque el primero y el tercero de sus miembros se contradicen y, por ello, con independencia del hecho de que también el primero y el segundo de sus miembros formen una contradicción. Podemos, por así decirlo, «cerrar nuestros ojos» a este último hecho y seguir teniendo la contradicción entre la conjunción de los primeros dos miembros y la negación del primer miembro.

Consideremos ahora la conjunción de que $A \& \sim A \& \sim B$. Esta es una contradicción. Es una contradicción porque la parte de ella formada por los primeros dos miembros «ya» es una contradicción. El carácter contradictorio de la conjunción de tres miembros depende del carácter contradictorio de la de dos miembros. Por tanto, no queremos decir que la conjunción de dos miembros implica la negación de que B. Dado que «B» representa una proposición arbitraria, deberíamos entonces tener que decir que una contradicción implica a cualquier proposición. Esta es la «paradoja» que queremos evitar.

${ }^{5}$ He presentado y tratado de defender esencialmente la misma explicación de la noción de implicación o consecuencia lógica en un temprano trabajo titulado «The Concept of Entailment» (en Logical Studies, London, Routledge \& Kegan Paul, 1957). Sin embargo, ya no estoy de acuerdo con todos los detalles de la argumentación en ese trabajo. 
Substituyamos «A» por «B». Que A\& A\& A es una contradicción. Dado que ello es ya así debido a que la conjunción de sus dos primeros miembros es contradictoria, ¿no deberíamos entonces decir que $\mathrm{A} \& \sim \mathrm{A}$ no implica que $\mathrm{A}$ ?

Que A\& A implica que A es un caso especial del principio según el cual una conjunción implica a cada uno de sus miembros. Pero, como una instancia de substitución de la contradicción que $A \& \sim A \& \sim B$, que $A \& \sim A$ no implica que $A$. Dos reglas para evaluar la implicación se intersectan, por así decirlo, en este punto, y podemos responder «Si» o «No» a la pregunta sobre si también una conjunción contradictoria implica a cada uno de sus miembros. Responder «Si» no importa comprometerse a admitir que «de una contradicción se sigue lógicamente cualquier proposición».

En virtud de argumentos simétricos se puede establecer la «ley de la implicación» según la cual una disyunción es implicada por cualquiera de sus miembros. Como un caso especial de ella tenemos que $A$ implica que $A \vee \sim A$, a pesar de que el carácter tautológico de $A \vee \sim A$ «ya» garantiza que la conjunción de que $A$ con la negación de que $A \vee \sim A$ sea una contradicción. El carácter tautológico de que $A \vee \sim A$ también garantiza que la conjunción de que $B$ con la negación de que $A \vee \sim A$ sea contradictoria. Esto, no obstante, no puede ser usado como una «prueba» de que una tautología es implicada por cualquier proposición. Porque la prueba de que $B \& \sim(A \vee \sim A)$ es inconsistente depende del hecho de que $\sim(A \vee \sim A)$ lo sea.

Espero que los ejemplos precedentes muestren con suficiente claridad cómo podemos «rescatar» la noción de implicación o consecuencia lógica de las «paradojas» de que una contradicción implica cualquier proposición y de que cualquier proposición implica cualquier tautología.

12. La idea de que una relación de implicación subsiste independientemente del valor de verdad o del valor modal de sus términos se refleja en la lectura condicional de esta relación (cfr. supra, sección 9). Decir «Si A entonces B» es afirmar un «vínculo» entre las dos proposiciones que se mantiene con independencia de si ellas resultan verdaderas o falsas, o aceptables o no. La aceptación de su implicación material como necesaria, i. e. el rechazo como inconsistente de la conjunción de una con la negación de la otra, es todo lo que importa. La implicación es una relación condicional lógicamente necesaria entre proposiciones. Por consiguiente, su aceptación no debe depender del previo rechazo de su antecedente ni de la previa aceptación de su consecuente. Pero esto no impide que, como cuestión de hecho, el antecedente sea rechazado como imposible, ni que el consecuente sea aceptado como necesario. 